\title{
CORRIGENDUM
}

\section{The neurodevelopmental model of schizophrenia: update 2005}

JC Rapoport, AM Addington and S Frangou

Molecular Psychiatry (2005) 10, 614. doi:10.1038/sj.mp.4001673

Correction to: Molecular Psychiatry (2005) 10, 439-449. doi:10.1038/sj.mp.4001642

Following the publication of this article, the authors discovered that 'MRC Psych' was included as an author by mistake. As listed above, there are only three authors: JL Rapoport, AM Addington and $S$ Frangou. 